\title{
Physics of Potassium Ion Channel Inactivation in Neurons
}

\author{
Ryan M. W. Collins \\ Department of Physics \\ University of Northern lowa \\ Cedar Falls, lowa 50614-0150 USA
}

Received: November 6, 2003

Accepted: March 5, 2004

\begin{abstract}
The electrical signaling capabilities of neurons depend on the flows of ions into and out of their axons. Potassium ions exit an axon's interior through a potassium channel or pore that connects the intracellular region with the extracellular region. The channel opens, or is activated, allowing potassium ions to exit. The channel then undergoes a blocking transition in which the channel is physically open but is blocked by some part of the larger channel molecule. This blocking process is called inactivation, and the physics by which it might occur forms the topic of our investigation. The $\mathrm{N}$-terminus region of the Drosophila shaker potassium ion channel was identified by Hoshi et al as having an important role in channel inactivation. Using the last 19 amino acids in the $\mathrm{N}$-terminus region, a mass and net charge were calculated. We investigated two forces that might affect the motion of this $\mathrm{N}$-terminus mass (tentatively identified as the blocking or inactivation particle): the magnetic field effects due to potassium ion current in the channel, and an electric force due to the decreasing density of potassium ions from the intracellular region. Time-of-flight calculations were calculated for the inactivation particle. These times will be discussed in terms of typical inactivation processes.
\end{abstract}

\section{INTRODUCTION}

A neuron is the type of cell responsible for information transfer in the body. The neuron (Figure 1) obtains information through its dendrites and then a signal, called an action potential, is sent along the axon to the terminal branches where it is passed to other cells. As the action potential travels down the axon, sodium ions flow into, and potassium ions out of, the axon. This transport happens through transmembrane channels that are selective of only one type of ion, i.e., $\mathrm{Na}^{+}, \mathrm{K}^{+}$ [1]. This paper focuses on the potassium ion channel.

The potassium ion channel is as a pore that allows potassium ions to pass from the inside to the outside of an axon. It is embedded in the cell wall which is made up of a bi-lipid membrane. The channel is constructed of a single large molecule composed of around 1800 amino acids linked together in a chain that weaves in and out of the axon membrane. The potassium ion channel molecule folds into four identical regions, each with six different transmembrane segments denoted S1 through S6 (see Figure 2). The ends of this molecule hang on the intracellular side of the membrane. One end is tipped with a nitrogen atom and is called the $\mathrm{N}$-terminus and the other has a carbon atom and is called the C-terminus, as shown in Figure 2 [1]. The so-called hydropathy index of its component amino acids determines how this molecule folds the way it does. Some amino acids are hydrophobic, meaning that they are repelled from water, while others are hydrophilic, meaning they are attracted to water. Because both the inside and outside

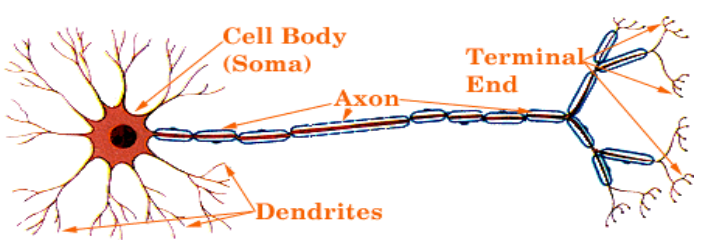

Figure 1. The basic structure of a neuron. 


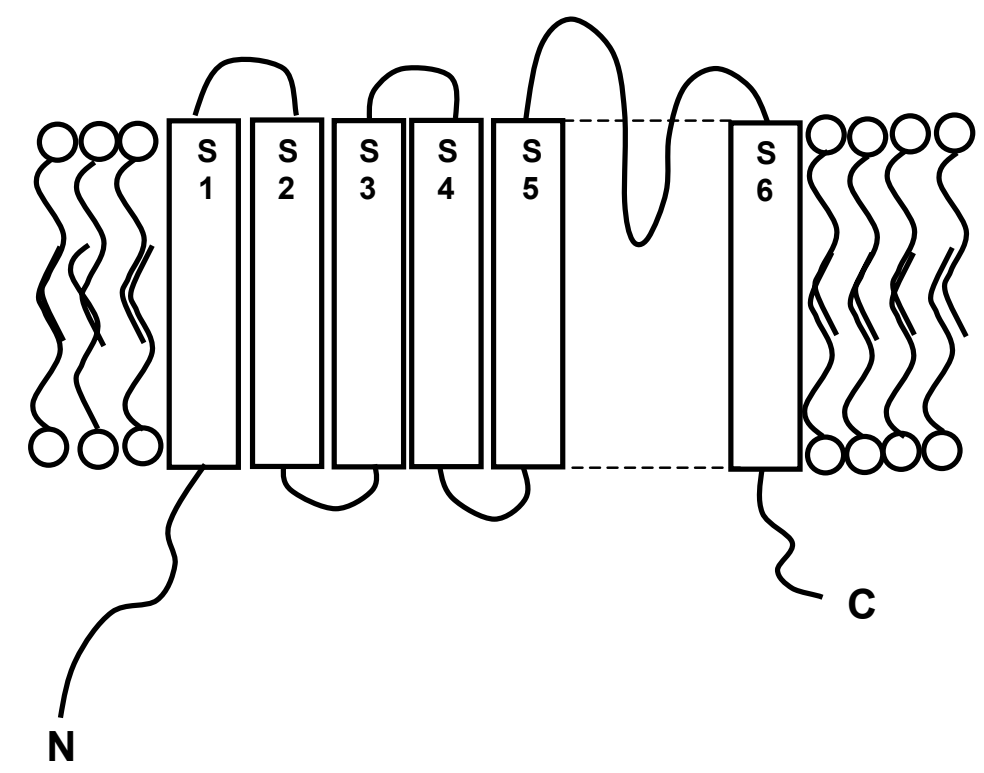

Figure 2. The secondary structure of the potassium channel molecule, showing one of four repeating sections. The channel molecule is inserted in a bilipid membrane, shown above from a side view, with the membrane unwrapped to show the transmembrane alpha-helical segments (S1-S6). The channel molecule weaves in and out of the bilipid membrane, with the N-terminus and $\mathrm{C}$-terminus sections in the intracellular region.

of the cell are in a saline solution, which is mostly water, this property determines how the channel molecule folds into its observed form. Hydrophobic portions of the molecule, in order to stay away from the saline solution, are sheltered inside the axon bilipid membrane, while hydrophilic amino acids are outside with the saline solution [1].

The potassium ion channel is more than simply a hole in the cell membrane through which ions can pass. The channel has three distinct states: activated; deactivated; or inactivated. Activated means that the channel is open to the flow of potassium ions through it; deactivated means the channel moves back to its resting state which no longer allows ions to flow; and inactivated which means the channel is open as in the activated state but is in addition physically blocked in some way so that ions cannot flow. There is fast inactivation, which happens one to three milliseconds after activation, and slow inactivation, which takes about six hundred milliseconds to occur [1, 2]. The topic of this research involves fast inactivation.

When an action potential passes over a potassium ion channel the S4 segment acts as a voltage sensor. Each S4 segment contains several charged amino acids that force the four S4 segments to rearrange in response to the electric fields of the action potential. In response to the S4 segments moving the S5 and S6 segments all four regions reconfigure so as to create an opening that allows $\mathrm{K}+$ ions to flow out of the cell. This process is what happens when it is said the channel activates. When the action potential goes back to equilibrium, the channel is deactivated, and the S4, S5, and $\mathrm{S} 6$ segments go back to their original positions [1].

When the channel is activated potassium ions flow through the channel in single file, separated by one water molecule [3], at a rate of approximately 6000 ions per millisecond.

\section{INACTIVATION OF THE POTASSIUM ION CHANNEL}

Experiments involving deletion mutations in the $\mathrm{N}$-terminus region of the Drosophila Shaker potassium ion channel found that the removal of charged amino acids in the first 19 residues, measured from the $\mathrm{N}$-terminus, slowed the fast inactivation of the potassium channel while the removal of the hydrophobic amino acids in the first 19 residues stopped fast inactivation. Large deletions from the molecule between residues 20 and 80 shortened the time taken 


\begin{tabular}{|l|l|l|l|l|l|l|l|l|l|l|l|l|l|l|l|l|l|l|l|}
\hline Number & 1 & 2 & 3 & 4 & 5 & 6 & 7 & 8 & 9 & 10 & 11 & 12 & 13 & 14 & 15 & 16 & 17 & 18 & 19 \\
\hline amino-acid & $\mathrm{M}$ & $\mathrm{A}$ & $\mathrm{A}$ & $\mathrm{V}$ & $\mathrm{A}$ & $\mathrm{G}$ & $\mathrm{L}$ & $\mathrm{Y}$ & $\mathrm{G}$ & $\mathrm{L}$ & $\mathrm{G}$ & $\mathrm{E}$ & $\mathrm{D}$ & $\mathrm{R}$ & $\mathrm{Q}$ & $\mathrm{H}$ & $\mathrm{R}$ & $\mathrm{K}$ & $\mathrm{K}$ \\
\hline type & $\mathrm{N}$ & $\mathrm{N}$ & $\mathrm{N}$ & $\mathrm{N}$ & $\mathrm{N}$ & $\mathrm{P}$ & $\mathrm{N}$ & $\mathrm{P}$ & $\mathrm{P}$ & $\mathrm{N}$ & $\mathrm{P}$ & - & - & + & $\mathrm{P}$ & $\mathrm{P}$ & + & + & + \\
\hline Hydropahy & 1.9 & 1.8 & 1.8 & 4.2 & 1.8 & -0.4 & 3.8 & -1.3 & -0.4 & 3.8 & -0.4 & -3.5 & -3.5 & -4.5 & -3.5 & -3.5 & -4.5 & -0.39 & -3.9 \\
\hline
\end{tabular}

Table 1. Characteristics of amino acids in the $\mathrm{N}$-terminus region.

for inactivation to occur [2]. This data fit very well with the ball and chain model originally proposed by Armstrong [4] to describe the inactivation of the sodium channel.

The ball and chain model, as shown in Figure 3, proposes that a "ball" is connected to the intracellular portion of the channel by a "chain". The ball moves into the channel and stops ion flow a few milliseconds after activation. The region consisting of the first 19 residues in the $\mathrm{N}$ terminus consists of a number of charged and hydrophobic amino acids. This area twists into a ball with the hydrophobic amino acids in the center of the structure and the charged amino acids on its exterior. The ball is attached to the rest of the channel molecule by a chain of approximately 60 amino acids [2].

The mass and charge for the $\mathrm{N}$ terminus region of the potassium ion channel molecule in Drosophila were determined by looking at the amino acid sequence of this region, given above. The mass and charge were used in equations of motion which were derived from Newton's $2^{\text {nd }}$ law and the Lorentz force law [5]. The amino acids are listed by their single-letter abbreviations [6], their types are listed as $\mathrm{N}$ for non-polar, P, for polar, "-“ for negatively charged, and "+" for positively charged, and for their hydropathy index [1]. In this project only the net charges of the amino acids are taken into account. The polar aspects are being ignored.

The hydropathy indexes given above indicate how each amino acid reacts with water. Those with positive indexes, with a maximum of +4.5 , are hydrophobic, meaning that they are repelled from water, while those with negative indexes, up to 4.5 , are hydrophilic, meaning they are attracted to water. Because the $\mathrm{N}$-terminus is surrounded by a saline solution the hydropathy index determines how the molecule folds to form a "ball". Hydrophobic portions of the molecule are inside the ball while the hydrophilic parts are on the outside.

\section{RESEARCH TOPICS AND MODEL CALCULATIONS}

The subject of this research was to investigate two interactions that might affect
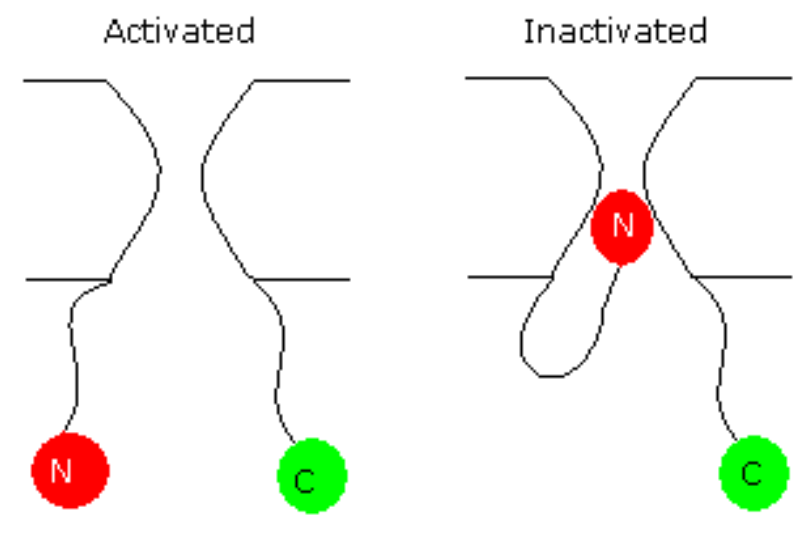

Figure 3. Ball and chain model of inactivation: the $\mathrm{N}$ terminus moves to block the channel. 
the motion of this $\mathrm{N}$-terminus mass: the magnetic field effects due to potassium ion current in the channel, and an electric force due to the decreasing density of potassium ions near the intracellular channel opening. Knowing the structure of the $\mathrm{N}$-terminus region of the potassium ion channel, and its relation to inactivation [2], gave a starting point for comparing experimental times for inactivation to occur with calculations of the motion of the $\mathrm{N}$-terminus ball to the opening of the channel because it allowed us to find the mass and net charge of the inactivation particle for use in motion equations. The first 19 amino acids in this region, as shown in Table 1, have known chemical compositions. Summing the mass of all atoms in this region gives a combined total of 2426 atomic mass units (amu), or $4.028 \times 10^{-24} \mathrm{~kg}$. The ball has a net of 2 amino acids, each with one elementary charge, giving a total charge for the ball of $3.206 \times 10^{-19} \mathrm{C}$. We estimate the length of the "chain," approximately 60 amino acids long, to be $200 \AA$.

\section{a. Magnetic Effects Due To Ionic Current Through Potassium Ion Channel}

We first investigated the effect of the magnetic force produced during activation due to the potassium ion current in the channel. For this computation the current of potassium ions through the channel was estimated as $6 \times 10^{5}$ ions per second [5]. The magnetic field due to the potassium ion current through the channel is treated as if it were a strait line segment with the current flowing in the negative $z$ direction as shown above. To find the magnetic field for this system, the equation for the magnetic field surrounding a straight current segment suffices [7],

$$
B=\frac{-\mu_{0} I}{4 \pi s}\left(\sin \theta_{2}-\sin \theta_{1}\right)
$$

where $\theta_{1}$ and $\theta_{2}$ are angles defined in Figure 4. A program compared the motion of the inactivation particle from rest at $60 \AA, 130 \AA$, and $200 \AA$ from the channel opening, due to a constant electric field to the motion of the ball due to the same constant electric field and the magnetic field caused by the current in the channel. This range of starting distances (60 to $200 \AA$ ) represents the "chain" in several states, from coiled to completely uncoiled. A constant electric field was used in the computation in place of a time and position varying electric field for simplicity and because the focus was to study the magnetic effects only. Using electric field magnitudes ranging from $1 \times 10^{4}$ $\mathrm{N} / \mathrm{C}$ to $1 \times 10^{7} \mathrm{~N} / \mathrm{C}$, the effect of the magnetic field was examined. Fields of these magnitudes were near the electric fields found in the next section. The initial position of the N-terminus "ball" was placed such that its displacement in the $z$ direction below the $x-y$ plane was equal to its distance from the $\mathrm{z}$ axis, s, so that $\sqrt{s^{2}+z^{2}}$ equaled $60 \AA$, $130 \AA$, or $200 \AA$, as appropriate.

As the results of Table 2 show, there is only a small change in the inactivation time due to the addition of the magnetic force. Hence the magnetic force due to the ionic current is not a primary force in determining the time needed for fast inactivation. As a note this computation does not take into account the magnetic field generated due to the opening from

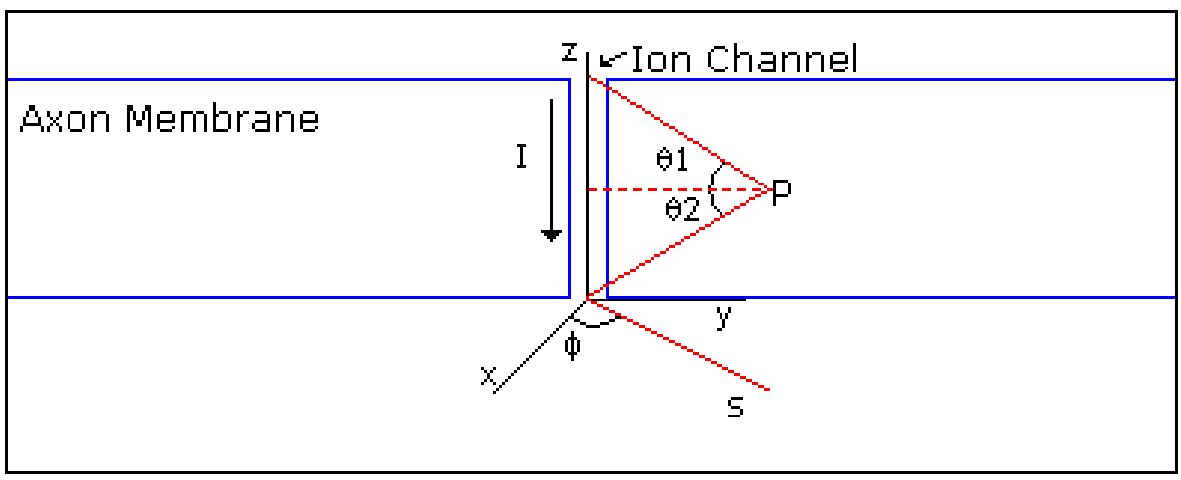

Figure 4. The coordinate axes and geometry used in the magnetic field calculation (§ III a). 


\begin{tabular}{|l|c|c|c|c|}
\hline \multirow{2}{*}{$\begin{array}{l}\text { Initial Displacement of the } \\
\text { N-terminus from the channel } \\
\text { opening }\end{array}$} & \multicolumn{4}{|c|}{ Electric Field Magnitudes (Newtons/Coulomb) } \\
\cline { 2 - 5 } & $1 \times 10^{4}$ & $1 \times 10^{5}$ & $1 \times 10^{6}$ & $1 \times 10^{7}$ \\
\hline $60 \AA$ & $1.17 \%$ & $1.17 \%$ & $1.17 \%$ & $1.21 \%$ \\
\hline $130 \AA$ & $0.54 \%$ & $0.54 \%$ & $0.54 \%$ & $0.55 \%$ \\
\hline $200 \AA$ & $0.35 \%$ & $0.36 \%$ & $0.35 \%$ & $0.36 \%$ \\
\hline
\end{tabular}

Table 2. The percentage increase in transit (i.e., inactivation) times of the N-terminus "ball" due to the inclusion of a magnetic term to the total force on the ball. The magnetic force can only divert the ball along its path, in a direction perpendicular to the ball's velocity and the local magnetic field vector. Equation (1) predicts a magnetic induction of order $10^{-12}$ Tesla [for $s=100$ $\AA$ ], so the small increase in transit times is no surprise.

opening from the surrounding saline solution and it is unknown what effects this field would have on the ball.

b. Electric Field Effects Due To The Decreasing Density Of $\mathrm{K}^{+}$lons Near The Intracellular Channel Opening

Before activation it is assumed that the potassium concentration is constant near the potassium ion channel opening. As potassium ions flow out of the axon through the ion channel, potassium ions surrounding the intracellular channel opening will diffuse toward the channel. This decreasing density of positive charges can be represented by an equal rate of accumulation of negative charges that diffuse from the opening with time into a charge-neutral environment. Spherical polar coordinates are used here with the origin at the intracellular channel opening (as shown in Figure 4).

To represent this diffusion of negative charge we start with the classical diffusion equation in one dimension [8]. If $Q$ is the amount of negative charge at $r=0$ at $t$ $=0$, then the concentration of charge is,

$$
c(r, 0)=Q \delta(r),
$$

where $c$ has units of negative particles per meter and where $\delta(r)$ is the Dirac delta function. The concentration of particles at a given distance $r$ at time $t$ is then,

$$
c(r, t)=\frac{Q e^{-r^{2} /(4 D t)}}{2(\pi D t)^{1 / 2}},
$$

for $t>0$, where $D$ is the diffusion constant of the negative charges in saline solution.

This equation gives the concentration of a set of charges placed at the origin at time equals zero. The flux of negative charges present in the case of the potassium ion channel requires a continuous input of charges. In order to arrive at this we integrate $c(r, t)$ over time and change $Q$ to $\Phi$ (measured in Coulombs per second). This change in parameter from $Q$ to $\Phi$ is done to ensure that $C(r, t)$ has units of Coulombs per meter.

$$
C(r, t)=\int_{0}^{t} c\left(r, t^{\prime}\right) d t^{\prime}=\int_{0}^{t} \frac{\Phi e^{-r^{2} /\left(4 D t^{\prime}\right)}}{2\left(\pi D t^{\prime}\right)^{1 / 2}} d t^{\prime}
$$

$$
C(r, t)=\frac{\Phi}{\sqrt{4 D \pi}} \int_{0}^{t} \frac{e^{-r^{2} /\left(4 D t^{\prime}\right)}}{\left(t^{\prime}\right)^{1 / 2}} d t^{\prime}
$$

First we do the following substitution, a

$$
\begin{aligned}
& \text { change of variable, as follows, } \\
& y=r^{2} / 4 D t^{\prime} \quad, d y=-r^{2} / 4 D t^{\prime 2} d t^{\prime}
\end{aligned}
$$

so that 
$t^{\prime}=\frac{r^{2}}{4 D y} ; d t^{\prime}=\frac{-t^{\prime 2} 4 D}{r^{2}} d y=\frac{-r^{2}}{4 D y^{2}} d y$

integral in y,

and thus,

$C(r, t)=\frac{\Phi r}{4 D \sqrt{\pi}} \int_{\frac{r^{2}}{4 D t}}^{\infty} \frac{e^{-y}}{y^{3 / 2}} d y$

Now, integrating by parts, we get another

$$
C(r, t)=\frac{\Phi r}{4 D \sqrt{\pi}}\left[\frac{2 e^{\frac{-r^{2}}{4 D t}}}{\sqrt{\frac{r^{2}}{4 D t}}}+\int_{\frac{r^{2}}{4 D t}}^{\infty} \frac{e^{-y}}{\sqrt{y}} d y\right] .
$$

Integrating, again by parts, produces

$$
C(r, t)=\frac{\Phi r}{4 D \sqrt{\pi}}\left[\frac{2 e^{\frac{-r^{2}}{4 D t}}}{\sqrt{\frac{r^{2}}{4 D t}}}+4 \sqrt{\frac{r^{2}}{4 D t}} e^{\frac{-r^{2}}{4 D t}}-4 \int_{\frac{r^{2}}{4 D t}}^{\infty} \sqrt{y} e^{-y} d y\right] .
$$

With $y=x^{2}$, such that $d y=2 x d x$,

$$
C(r, t)=\frac{\Phi r}{4 D \sqrt{\pi}}\left[\frac{2 e^{\frac{-r^{2}}{4 D t}}}{\sqrt{\frac{r^{2}}{4 D t}}}+4 \sqrt{\frac{r^{2}}{4 D t}} e^{\frac{-r^{2}}{4 D t}}-4 \int_{\sqrt{\frac{r^{2}}{4 D t}}}^{\infty} x(2 x) e^{-x^{2}} d x\right] .
$$

Now, yet again, we do integration by parts

with $\mathrm{u} \equiv \mathrm{x}$ and $\mathrm{dv} \equiv-2 \mathrm{x} \exp \left(-\mathrm{x}^{2}\right)$, to get

$$
-4 \int_{\sqrt{\frac{r^{2}}{4 D t}}}^{\infty} x(2 x) e^{-x^{2}} d x=4 \sqrt{\frac{r^{2}}{4 D t}} e^{\frac{-r^{2}}{4 D t}}-4 \int_{\sqrt{\frac{r^{2}}{4 D t}}}^{\infty} e^{-x^{2}} d x
$$

so that

$$
C(r, t)=\frac{\Phi r}{4 D \sqrt{\pi}}\left[\frac{2 e^{\frac{-r^{2}}{4 D t}}}{\sqrt{\frac{r^{2}}{4 D t}}}+4 \sqrt{\frac{r^{2}}{4 D t}} e^{\frac{-r^{2}}{4 D t}}+4 \sqrt{\frac{r^{2}}{4 D t}} e^{\frac{-r^{2}}{4 D t}}-4 \int_{\sqrt{\frac{r^{2}}{4 D t}}}^{\infty} e^{-x^{2}} d x\right]
$$

Now,

$$
\int_{\sqrt{\frac{r^{2}}{4 D t}}}^{\infty} e^{-x^{2}} d x=\int_{0}^{\infty} e^{-x^{2}} d x-\int_{0}^{\sqrt{\frac{r^{2}}{4 D t}}} e^{-x^{2}} d x
$$

whose integrals can be evaluated using the standard literature. Integrals 3.321 .2 and
3.321.3 of Gradshteyn and Ryzhik [9] tell us that

$$
\int_{0}^{\infty} e^{-x^{2}} d x=\frac{\sqrt{\pi}}{2}
$$

and that 


$$
\int_{0}^{\sqrt{\frac{r^{2}}{4 D t}}} e^{-x^{2}} d x=e^{-r^{2} / 4 D t} \sum_{k=0}^{\infty} \frac{\left(2^{k}\left(\frac{r}{\sqrt{4 D t}}\right)^{2 k+1}\right)}{(2 k+1) ! !}
$$

Thus,

$$
C(r, t)=\frac{\Phi r}{4 D \sqrt{\pi}}\left[\frac{2 e^{\frac{-r^{2}}{4 D t}}}{\sqrt{\frac{r^{2}}{4 D t}}}+8 \sqrt{\frac{r^{2}}{4 D t}} e^{\frac{-r^{2}}{4 D t}}-2 \sqrt{\pi}+4 e^{-r^{2} / 4 D t} \sum_{k=0}^{\infty} \frac{\left(2^{k}\left(\frac{r}{\sqrt{4 D t}}\right)^{2 k+1}\right)}{(2 k+1) ! !}\right] .
$$

So, finally, the concentration of effective negative charge is

$$
C(r, t)=\frac{\Phi}{4 \sqrt{\pi} D}\left[4 \sqrt{D t} e^{-r^{2} / 4 D t}+\frac{4 r^{2} e^{\frac{-r^{2}}{4 D t}}}{\sqrt{D t}}-2 \sqrt{\pi} r+4 r e^{-r^{2} / 4 D t} \sum_{k=0}^{\infty} \frac{\left(2^{k}\left(\frac{r}{\sqrt{4 D t}}\right)^{2 k+1}\right)}{(2 k+1) ! !}\right]
$$

for $\mathrm{t}>0$.

This is the one dimensional diffusion equation for a constant flux at the origin. In order to change this to represent the three dimensional interior of the axon it will be multiplied by a correction factor $G$, where

$$
G(r) \equiv \frac{1}{r^{2}}
$$

There also should be some constant attached to $G$, but $D$, the diffusion constant, which we must approximate in any case, will effectively absorb this constant. This correction factor was chosen because it gives the concentration dimensional consistency for three dimensions, simplifies future computations, and corrects for the $r^{2}$ term encountered when moving radially outward on a hemisphere. In order ensure that $G$ remains a reasonable correction factor, it is important to assume that the inactivation particle stops before $r=0$. So for the purpose of this computation, the equations of motion will only be examined up to $1 \AA$ from the channel rather than $0 \AA$. This is a reasonable limitation to apply because of the size of the inactivation particle is greater than one angstrom, thus it will hit the channel mouth at a greater center-of-mass radius than $0 \AA$. From $\mathrm{C}(\mathrm{r}, \mathrm{t})$, with $G(r)$, the electric field due to the relative concentration of negative charges near the intracellular mouth of the channel can be found.

To simplify the computations in finding the force Gauss's law was used for a sphere centered on the channel opening with the ball on its surface. This will cause the predicted time to be somewhat greater than otherwise.

Applying Gauss' Law [5] to find the intracellular electric field, as a function of radial distance $r$ and time $t$, 


$$
E(R, t)=\frac{1}{4 \pi \varepsilon_{0} r^{2}} \int_{0}^{R} 4 \pi r{ }^{2} C(r, t) * G(r) d r
$$

or,

$$
E(R, t)=\frac{\Phi}{4 \varepsilon_{0} D \sqrt{\pi}} \int_{0}^{R}\left[4 \sqrt{D t} e^{-r^{2} / 4 D t}+\frac{4 r^{2} e^{\frac{-r^{2}}{4 D t}}}{\sqrt{D t}}-2 \sqrt{\pi} r+4 r e^{-r^{2} / 4 D t} \sum_{k=0}^{\infty} \frac{\left(2^{k}\left(\frac{r}{\sqrt{4 D t}}\right)^{2 k+1}\right)}{(2 k+1) ! !}\right] d r
$$

In the integration over dr each of the above terms with an $\exp \left(-r^{2}\right)$ involves a summation in the solution. Each of these summations truncates very quickly. We thus have approximated them by their lowest order first non-zero terms. In addition the summation over $\mathrm{k}$ in the fourth term also truncates very quickly and thus it too is approximated in lowest order. The integrals for the first and third terms are fairly strait forward, however the second and fourth term required the following substitution in order to remove the $r^{2}$ in front of the exponential. With this substitution the results follows relatively quickly.

$$
\frac{\partial}{\partial a}\left(e^{-a r^{2}}\right)=-r^{2} e^{-a r^{2}}, \text { where } a=\frac{1}{4 D t}
$$

Once the all four terms are solved the result becomes,

$$
E(R, t)=\frac{2 \Phi t e^{-R^{2} / r D t}}{R \varepsilon_{0} \sqrt{4 D t \pi}}+\frac{\Phi R}{3 \varepsilon_{0} D \sqrt{D t \pi}}+\frac{\Phi}{R D \varepsilon_{0}}+\frac{\Phi R}{3 \varepsilon_{0} D \sqrt{4 D t \pi}}
$$

in magnitude, with the direction radially inward toward the intracellular mouth of the channel.

From this the force, and hence the acceleration, on the ball can be found using Newton's second law,

$$
a_{\text {Ball }}=\frac{Q_{\text {Ball }} \cdot E(R, t)}{M_{\text {BALL }}}
$$

A program was written to calculate $E(R, t)$ on the ball at small time steps, and then numerically find the time required for the ball to reach the channel opening. Euler's method was employed [10]. The initial conditions of for the N-terminus "ball" and its environment are given in Table 3 .

According to the computations the $\mathrm{N}$-terminus ball will reach the channel opening at about $7 \times 10^{-9}$ seconds. The normal inactivation speed is around $10^{-3}$ seconds, a speed far faster than happens experimentally [11].
A number of qualities of the system could be implemented to correct for much of the discrepancy between the real and the computed times. The saline solution surrounding the $\mathrm{N}$-terminus chain will cause

\begin{tabular}{|l|c|}
\hline $\begin{array}{c}\text { N-terminus } \\
\text { parameters and initial } \\
\text { conditions }\end{array}$ & Value \\
\hline N-terminus mass & $4.0 \times 10^{-24} \mathrm{~kg}$ \\
\hline N-terminus net charge & $3.206 \times 10^{-19} \mathrm{C}$ \\
\hline Current I & $-9.62 \times 10^{-13} \mathrm{C} / \mathrm{s}$ \\
\hline $\begin{array}{l}\text { Initial displacement, } \\
\mathrm{R}_{0}\end{array}$ & $2 \times 10^{-8} \mathrm{~m}$ \\
\hline Initial "ball" velocity & $0 \mathrm{~m} / \mathrm{s}$ \\
\hline $\begin{array}{l}\text { Initial "ball" } \\
\text { acceleration }\end{array}$ & $0 \mathrm{~m} / \mathrm{s}^{2}$ \\
\hline Diffusion constant, D & $1 \times 10^{-4} \mathrm{~m}^{2} / \mathrm{s}$ \\
\hline
\end{tabular}

Table 3. N-terminus values. 
a drag on the ball as it travels to the channel opening, hence slowing it down. The mass used for the $\mathrm{N}$-terminus region only included the mass of the last 19 amino acids on the chain, and not the mass of the entire chain. The chain has about 60 amino acids, in addition to the 19 in the ball and some of this mass will be drug along by the ball. This will increase the effective mass of the ball and the amount of resistance the ballchain encounters as it moves through the saline solution. A dense concentration of negative charge, which is effectively what is seen near the channel opening during this process, will disperse more quickly due to the repulsive forces of the surrounding charge, hence it is possible that the real diffusion rate of the charges could be much greater than estimated. This would cause the number of charges with in the Gaussian surface to decrease and in turn decrease the force felt by the ball. The shielding properties of the saline solution to the electric field were also not taken into account.

\section{CONCLUSIONS}

The effects due to the magnetic field created by the ionic currents through the potassium ion channel were found to decrease the time for inactivation to occur, but only by a very small amount. Overall this magnetic field only reduced the inactivation time by around one percent. This shows that this is not a primary force in this system compared to the force created by the decreasing density of positively charged potassium ions near the channel opening.

The effects due to the electric field created by the decreasing density of positively charged potassium ions near the channel opening have been shown to be very large. The results of this project show that with our given assumptions, and without any interposing obstacles to the $\mathrm{N}$-terminus ball, this force would cause inactivation about $10^{6}$ times faster than measured in real potassium ion channels. This suggests that this electrically induced force could be the primary driving force responsible for pushing the $\mathrm{N}$-terminus ball to the channel opening, although other forces responsible for the damping of the $\mathrm{N}$-terminus motion need to be explored to bring computational inactivation times in line with observed times.

There are several possibilities for future research in the area of the physics of potassium ion channel inactivation. Motion due to the magnetic field due to the movement of potassium ions toward the channel opening, the damping/frictional force created by the presence of the saline solution surrounding the $\mathrm{N}$-terminus region, and the electric and magnetic shielding due to the saline solution are all ways to try to correct the fields computed above. Finally, improving mathematical simplifications used to obtain these results would also increase the accuracy of the computations.

\section{REFERENCES}

1. David J. Aidley, The Physiology of Excitable Cells, 4th edn. (Cambridge University Press, Cambridge, UK, 1998).

2. T. Hoshi, W. N. Zagotta, and R. W Aldrich, Science 250 (1990) pp. 533538.

3. Christopher Miller, See potassium run, Nature 414 (2001) pp. 23-24.

4. Armstrong, C. M. (1981) Sodium channels and gating currents, Physiol. Rev. 61 (1981) pp. 644-83.

5. Single-letter abbreviations for the amino acid residues are: A, Ala; C, Cys; D, Asp; E, Glu; F, Phe; G, Gly; H, His; I, Ile; K, Lys; L, Leu; M, Met; N, Asn; P, Pro; Q, GIn; R, Arg; S, Ser; T, Thr; V, Val; W, Trp, and $\mathrm{Y}$, Try.

6. Russell K. Hobbie, Intermediate Physics for Medicine and Biology, $3^{\text {rd }}$ edn (Springer-Verlag, AIP Press, New York, 1997) p. 138.

7. D. J. Griffiths, Introduction to Electrodynamics, $3^{\text {rd }}$ edn (Prentice-Hall International, Upper Saddle River, New Jersey, USA, 1999) p. 217.

8. J. D. Murray, Mathematical Biology (Springer-Verlag, New York, 1989) pp 232-236.

9. I. S. Gradshteyn and I. M. Ryzhik, Table of Integrals, Series, and Products (Academic Press, New York, 1965).

10. Paul L. DeVries, A First Course in Computational Physics (John Wiley \& Sons, New York, 1994), p.208.

11. W. N. Zagotta, T. Hoshi, and R. W Aldrich, Science 250 (1990) pp 568-571. 


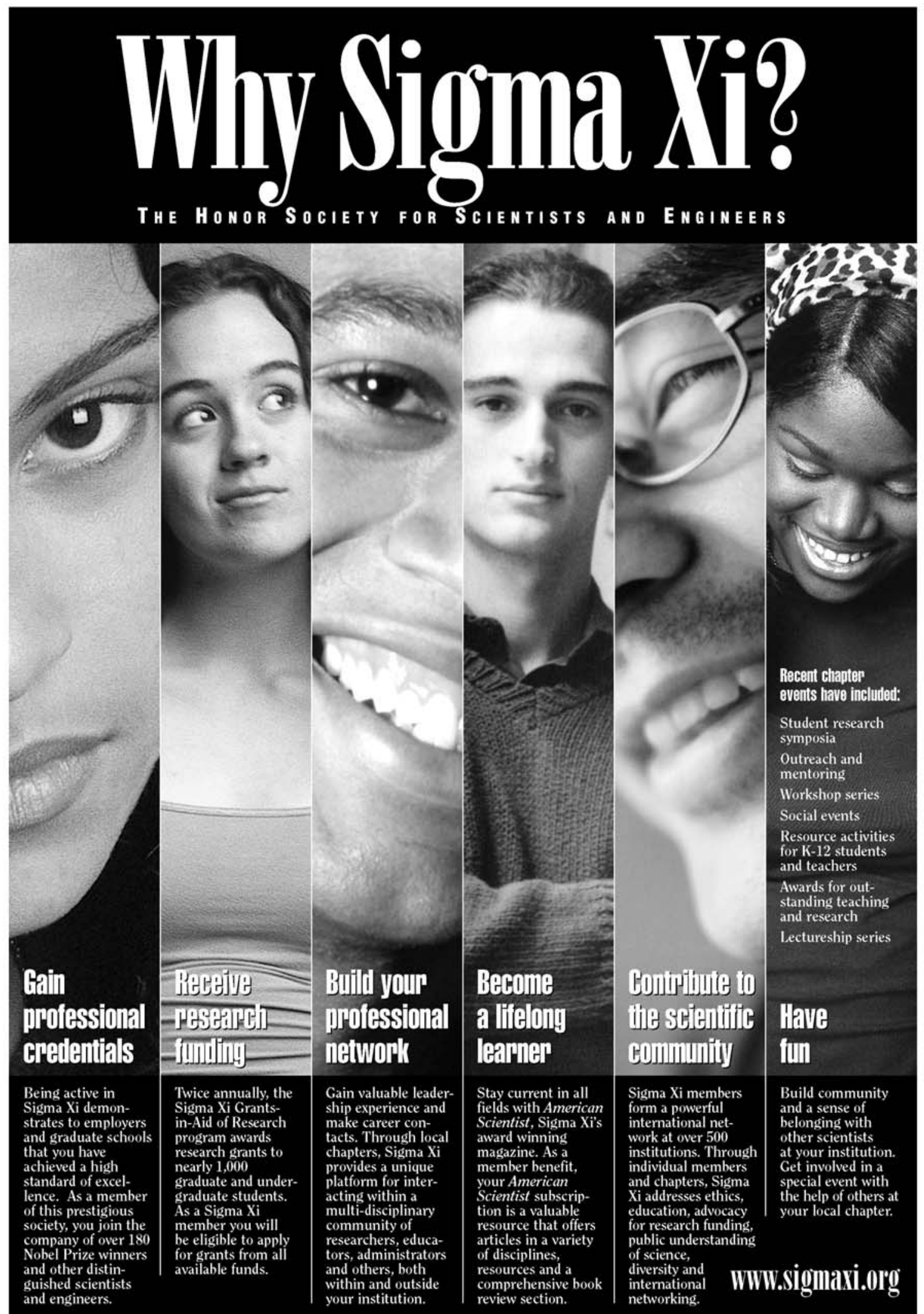

Sigma Xi, The Seientific Research Society • 98 Alexander Drive • P.O. Box 13975 • Research Triangle Park, NC 27708 • 818-549-4691 • 800-243-6534 